\title{
INTERAÇÃO DE TARTARUGAS MARINHAS E A PESGA NO BRASIL: UMA REVISÃO DA LITERATURA
}

\author{
Interaction of sea turtle and Brazilian fisheries: \\ a literature review
}

\author{
Talita Ribeiro Gagliardi ${ }^{1}$, Thaís Cândido Lopes ${ }^{1}$, Thiago Zagonel Serafini ${ }^{1}$ \\ 1 Departamento de Ciências do Mar (DCMar), Universidade Federal de São Paulo (UNIFESP), \\ Campus Baixada Santista, talitagagliardi@gmail.com@gmail.com
}

\begin{abstract}
RESUMO
A pesca no Brasil é bastante diversificada, abrangendo comunidades pesqueiras artesanais distribuídas ao longo da costa, além de frotas industriais. Das sete espécies de tartarugas marinhas que ocorrem ao redor do mundo, cinco são encontradas no Brasil e todas interagem com a pesca. Esforços de pesquisa e conservação visando mitigar a captura incidental (bycatch) das tartarugas vem sendo realizados, sendo considerada uma linha de ação prioritária para a pesquisa, manejo e conservação. Com o objetivo de compreender de que maneira a pesquisa vem lidando com a questão da interação das tartarugas marinhas com a pesca no Brasil, no presente trabalho realizamos uma revisão da literatura visando identificar os avanços e as lacunas do conhecimento sobre a temática no país. Foi identificada uma literatura crescente desde os anos de 1960, com um aumento mais expressivo a partir dos anos 2000. Apesar do aumento das pesquisas, observamos um predomínio da literatura sobre a interação com a pesca industrial, em especial o espinhel pelágico, em detrimento de pescarias artesanais, a qual ainda carece de estudos, principalmente nas regiões norte e nordeste. Ainda, se faz necessário um melhor dimensionamento da mortalidade ocasionada pela captura na pesca e seu impacto nas populações, assim como na ampliação das estratégias de conservação.
\end{abstract}

Palavras-chave: captura incidental, pesca artesanal, pesca industrial, conservação, Brasil.

Recebido em: 26/09/2017

Aprovado em: 30/05/2018

Publicado online em: 05/09/2018 


\section{ABSTRACT}

Fishing in Brazil is highly diversified, encompassing small-scale fishery communities along the coast, as well as industrial fleets. Of the seven species of sea turtles around the world, five are found in Brazil and all interact with fishing. Research and conservation efforts to mitigate bycatch of sea turtles have been carried out, being considered a priority for research, management and conservation. In order to understand how the research has dealt with the question of the interaction of sea turtles with fishing in Brazil, in the present study we carry out a review of the literature aiming to identify the advances and gaps of the knowledge on the subject in Brazil. A growing literature has been identified since the 1960s, with a more significant increase since the 2000s. Despite the increase in research, we have observed a predominance of the literature on interaction with industrial fishing, especially pelagic longline, to the detriment of small-scale fisheries, which still lacks studies, mainly in the north and northeast coast. Still, it is necessary a better dimensioning of the mortality caused by the capture in the fishery and its impact on the populations, as well as in the expansion of the conservation strategies.

Keywords: bycatch, small-scale fishery; industrial fishery; conservation, Brazil.

\section{INTRODUÇÃO}

Existem no mundo sete espécies de tartarugas marinhas e todas estão catalogadas pela União Internacional para Conservação da Natureza (IUCN) como ameaçadas ou criticamente ameaçadas de extinção (IUCN, 2013). Cinco espécies estão presentes no Brasil (Caretta caretta, Chelonia mydas, Eretmochelys imbricata, Lepidochelys olivacea e Dermochelys coriacea). A cabeçuda (C. caretta) é abundante em áreas de desova principalmente nos estados do Rio de Janeiro, Espírito Santo, Bahia e Sergipe. A de-pente (E. imbricata) é encontrada em áreas de desova no nordeste brasileiro, principalmente na Bahia, e a oliva (L. olivacea) no litoral norte da Bahia e Sergipe. A de-couro (D. coriacea) está presente em áreas de desova apenas no litoral do Espírito Santo. Jovens e adultos das espécies podem ser encontrados em áreas de alimentação na costa e águas oceânicas, com distribuição e abundância diferente conforme a espécie e seu ciclo de vida (Marcovaldi \& Marcovaldi, 1999).

A interação de tartarugas marinhas com a pesca é considerada uma das principais ameaças às populações de todas as espécies no mundo (NRC, 1990; Hamann et al., 2010). No Brasil, esforços de conservação e pesquisa se iniciaram especialmente nos anos de 1980, voltados para a redução de ameaças em áreas de reprodução (Marcovaldi \& Marcovaldi, 1999). Após décadas de ações de conservação, as populações das espécies no Brasil vêm demonstrando indícios de recuperação nas áreas de desova (Marcovaldi \& Chaloupka, 2007; Marcovaldi et al., 2007; Silva et al., 2007; Thomé et al., 2007). Por outro lado, a captura incidental na pesca é crescente, se constituindo hoje como a principal ameaça para a manutenção das populações, o que tem resultado em esforços de pesquisa e ação, principalmente a partir dos anos de 1990 (Marcovaldi et al., 1998; Marcovaldi et al., 2002).

A captura de tartarugas marinhas tem sido reportada tanto em pescarias industriais quanto artesanais. Na pesca industrial, o espinhel pelágico e as redes de deriva se destacam com as pescarias de maior potencial de interação (Sales et al., 2008; Fiedler et al., 2012). Na pesca artesanal, as capturas abrangem uma diversidade de pescarias, que variam em suas 
características ao longo da costa (Marcovaldi et al., 2006). A estimativa de mortalidade decorrente da interação ainda carece de uma avaliação mais precisa, porém encalhes de praia vem sendo utilizados como indicadores de mortalidade pela pesca (Silva et al., 2002; Soto et al., 2002; Guebert et al., 2005; Lima \& Melo, 2005; Castilhos \& Tiwari, 2006; Monteiro et al. 2016). As estratégias de mitigação do impacto da pesca vêm ocorrendo através do desenvolvimento de alternativas tecnológicas, como por exemplo o uso de anzol circular na pesca de espinhel pelágico (Sales et al. 2010), e ações de conservação, tais como educação e conscientização ambiental (Marcovaldi et al., 2002).

Em 2010, Hamann e colaboradores sugeriram cinco categorias prioritárias de pesquisa, que se desdobram em 20 questões-chave, para o manejo e conservação das tartarugas marinhas, com base na avaliação de pesquisadores ao redor do mundo. Na categoria de ameaças, uma das meta-questões diz respeito à interação das tartarugas marinhas com a pesca, sendo esta: "Quais são os principais fatores de captura incidental e como podem ser mitigados de uma maneira viável em termos ecológicos, econômicos e sociais?". Mais recentemente, Rees et al. (2016) realizaram uma revisão bibliográfica para avaliar como as pesquisas tem focado nestas questões-chave para a conservação das tartarugas marinhas globalmente. No sentido de compreender o desenvolvimento da pesquisa com a questão da interação das tartarugas marinhas e a pesca no Brasil, realizamos uma revisão da literatura visando identificar os avanços e as lacunas do conhecimento sobre a temática no país. Espera-se contribuir para uma visão da produção científica sobre esta temática, que é de grande interesse para a conservação das tartarugas marinhas no Brasil e no mundo, possibilitando identificar áreas que necessitam de maiores esforços de pesquisa.

\section{MATERIAIS E METODOS}

Para a obtenção da literatura, foram realizadas buscas nas bases de dados da Web of Science, Scopus, Google Scholar e a base de periódicos CAPES. As palavras-chaves utilizadas foram sea turtle, bycatch, beach stranding, fisheries interaction, Brazil (língua inglesa) e pesca, interação com pesca, captura incidental, encalhes, tartarugas marinhas, Brasil (vernácula). Também foram verificadas as referências citadas nos estudos identificados para localizar eventuais artigos que não surgiram na pesquisa nas bases de dados bibliográficas. Nessas condições, foram selecionadas e reunidas literaturas específicas publicadas sobre a temática captura incidental e pesca. Como critério de inclusão, foram considerados somente artigos publicados em periódicos nacionais e internacionais com ISSN e que abordavam algum aspecto relacionado à interação de tartarugas marinhas e pesca. Os artigos encontrados que estavam de acordo com os objetivos propostos foram organizados em tabelas, incluindo informações como: ano de publicação; localização geográfica; espécies mencionadas; tipos de pescarias envolvidas; medidas de mitigação; e dados de captura, incluindo Captura por Unidade de Esforço (CPUE). Artigos que tratavam tanto da pesca artesanal quanto industrial foram contabilizados para ambos as categorias de pesca.

\section{RESULTADOS}

A partir da pesquisa bibliográfica foram selecionados trabalhos publicados no período de 1965 a 2016. Foram identificados 602 artigos relacionados às palavras-chave e 68 atenderam aos critérios de seleção, dos quais 40 foram selecionados (Apêndice 1) por estarem de acordo com os objetivos propostos. Dos 40 artigos encontrados, 28 foram pub- 
licados em inglês, oito em português e quatro em espanhol, sendo $80 \%$ das publicações em revistas internacionais e $20 \%$ em revistas nacionais. As revistas que mais tiveram publicações foram a Collective Volume of Scientific Papers ICCAT e a Marine Turtle Newsletter, com seis artigos publicados em cada. A publicação mais antiga levantada foi de 1965 . O número de publicações se torna mais expressivo somente ao final dos anos de 1990 e início de 2000, quando então segue uma tendência de crescimento contínuo, tanto para a pesca artesanal quanto industrial, porém aparentemente maior na pesca artesanal, apesar do atual menor número de artigos desta última (Figura 1). Parte dos artigos tratam tanto de pesca artesanal quanto industrial.

Figura 1 - Evolução da produção científica (número de artigos) sobre a interação de tartarugas marinhas e a pesca industrial e artesanal entre 1965 e 2016.

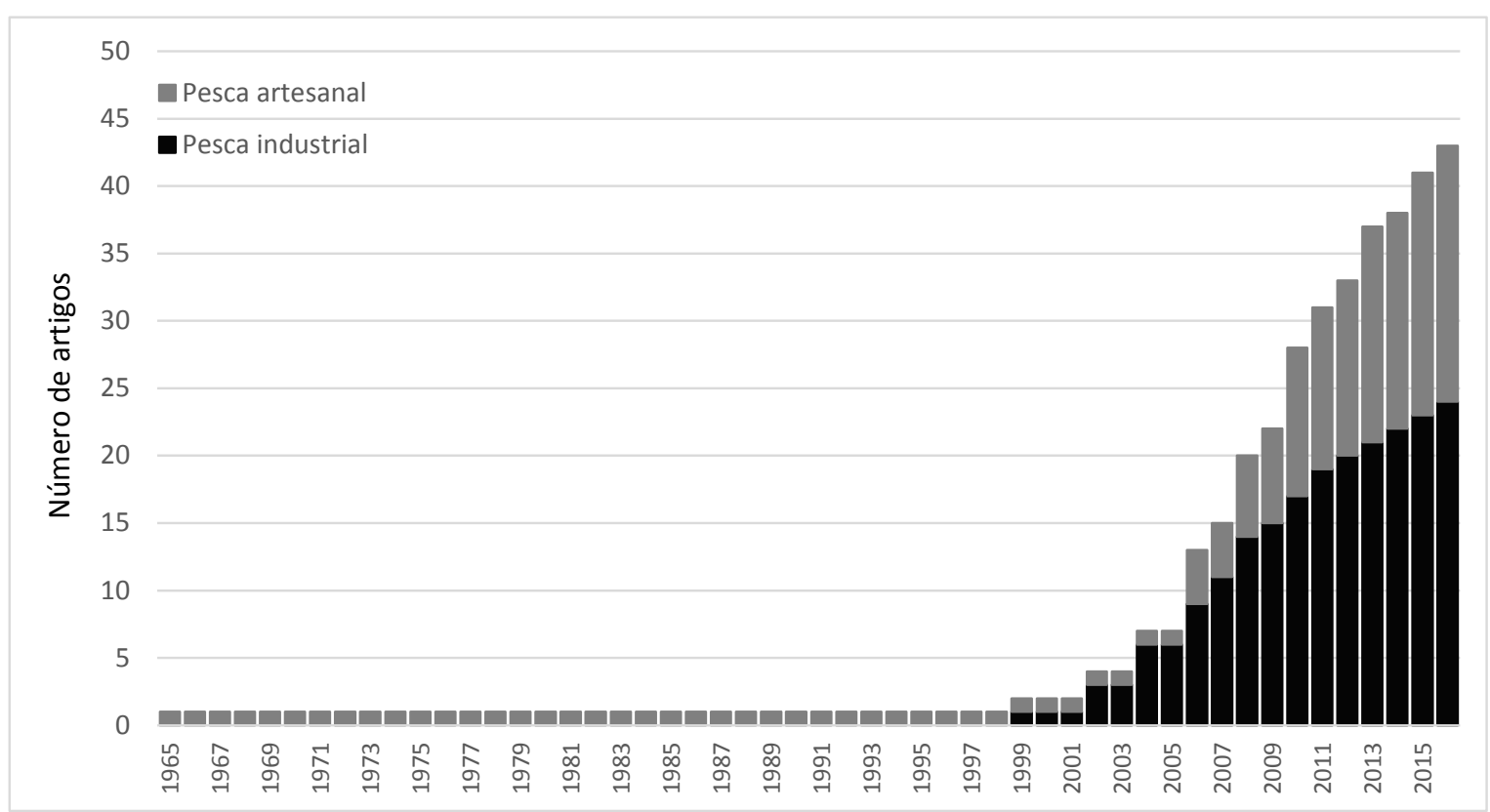

Atualmente, há um predomínio da produção científica com relação à interação em águas oceânicas ao longo da costa brasileira. Com relação aos estados, São Paulo se destaca com $15 \%$ dos trabalhos, em oposição aos estados da região norte e nordeste do país, os quais, conjuntamente, estão relacionados a 22,5\% dos trabalhos. A espécie mais presente nos estudos foi a C. caretta (30 artigos), seguida por D. coriacea (26 artigos), C. mydas (19 artigos), L. olivacea (15 artigos) e E. imbricata (13 artigos) (Figura 2).

A Tabela I sintetiza as informações dos trabalhos que reportaram dados de captura. Foram identificadas oito pescarias industriais que interagiram com tartarugas marinhas, principalmente o espinhel pelágico (pelagic longline) (57,5\% dos artigos). No caso das pescarias artesanais, foram identificadas as de redes de emalhe (de diferentes especificações com base no petrecho e/ou espécies-alvo) (27,5\% dos artigos), cerco-fixo, cercoflutuante, arrasto de fundo, linha-de-mão, espinhel e arrasto de praia. Apenas 57,5\% da literatura apresentou dados de captura a partir do monitoramento da pesca. Já os dados de Captura por Unidade de Esforço (CPUE) foram escassos, presentes apenas em 37,5\% dos estudos. 
Figura 2. Produção científica (número de artigos) por localização geográfica (barras) e percentual por espécies (gráfico pizza). $\mathrm{CC}=$ Caretta caretta, $\mathrm{DC}=$ Dermochelys imbricata, $\mathrm{CM}=$ Chelonia mydas, $\mathrm{LO}=$ Lepidochelys olivacea, $\mathrm{EI}=$ Eretmochelys imbricata.

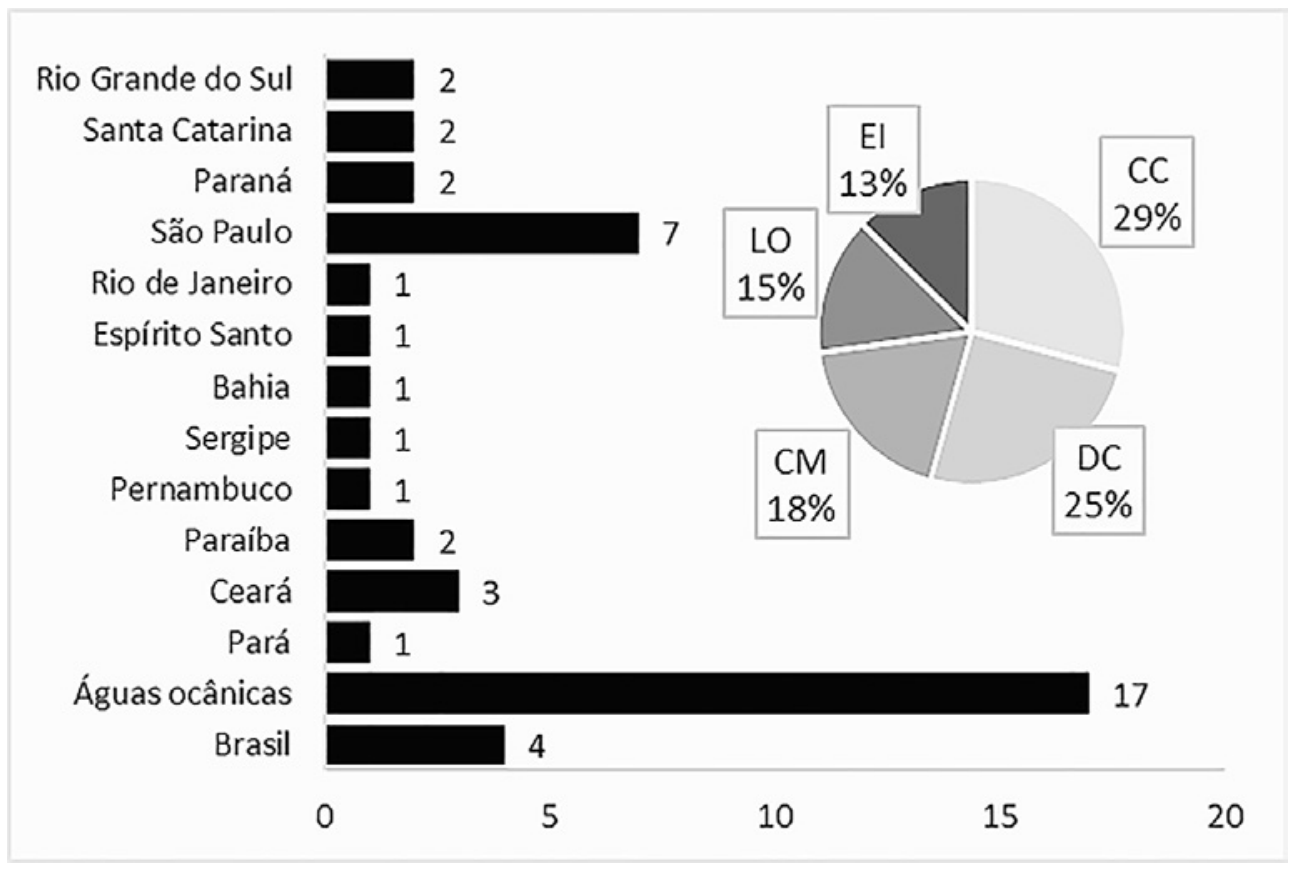

A Tabela II apresenta medidas mitigadoras e planos/ações propostos para lidar com a interação das tartarugas marinhas e a pesca, com base na literatura. Em geral, as ações mais frequentemente sugeridas, foram relacionadas ao monitoramento das áreas de incidência das tartarugas, pesquisa e sensibilização da população.

Tabela I - Dados de captura incidental de tartarugas marinhas por pescarias, região e espécies capturadas no Brasil. DC $=$ Dermochelys coriacea $; \mathrm{CC}=$ Caretta caretta $; \mathrm{LO}=$ Lepidochelys olivacea $; \mathrm{CM}=$ Chelonia mydas; $\mathrm{EI}=$ Eretmochelys imbricata $; \mathrm{BR}=$ Costa Brasileira; $\mathrm{CE}=$ Ceará; $\mathrm{OC}=$ Águas Oceânicas; $\mathrm{PR}=$ Paraná, $\mathrm{RJ}=$ Rio de Janeiro, RS = Rio Grande do Sul, $\mathrm{SP}=\mathrm{São}$ Paulo. $\mathrm{CCC}=$ Comprimento Curvilíneo de Carapaça. $\mathrm{CPUE}=$ Captura por Unidade de Esforço ${ }^{1}$.

\begin{tabular}{|c|c|c|c|c|c|}
\hline Referência $^{2}$ & Espécie & $\begin{array}{c}\text { Pescaria } \\
\text { industrial }\end{array}$ & $\begin{array}{l}\text { Pescaria } \\
\text { artesanal }\end{array}$ & $\begin{array}{l}\text { Estado/ } \\
\text { região }\end{array}$ & Dados de captura \\
\hline Paiva \& Nomura (1995) & $\mathrm{CM}$ & - & Cerco-Fixo & $\mathrm{CE}$ & $\begin{array}{l}\text { De } 1962 \text { a } 1965 \text { foram capturados } \\
963 \mathrm{CM} \text {. }\end{array}$ \\
\hline Sampaio et al. (1999) & DC & $\begin{array}{l}\text { Espinhel } \\
\text { pelágico }\end{array}$ & - & OC & $\begin{array}{l}\text { Barco espinheleiro com equipamento de } \\
28.000 \text { m e } 600 \text { anzóis capturou } 2 \text { DC. }\end{array}$ \\
\hline Kotas et al. (2004) & $\mathrm{CC} / \mathrm{DC}$ & $\begin{array}{l}\text { Espinhel } \\
\text { pelágico }\end{array}$ & - & OC & $\begin{array}{l}\text { Monitoramento de três viagens de } \\
\text { pesca em } 1998 \text { a bordo de embarcações } \\
\text { da frota pesqueira sediada em Itajaí } \\
\text { (SC). Foram capturadas } 145 \text { CC (CCC } \\
\text { entre } 46 \text { e } 73 \mathrm{~cm} \text { ) e } 20 \text { DC, com uma } \\
\text { CPUE de } 4,31 \text { e } 0,59 \text {, respectivamente. }\end{array}$ \\
\hline Lewison et al. (2004) & $\mathrm{CC} / \mathrm{DC}$ & $\begin{array}{l}\text { Espinhel } \\
\text { pelágico }\end{array}$ & - & OC & $\begin{array}{l}\text { Análise de dados de captura reportados } \\
\text { para comissões internacionais de pesca } \\
\text { referente ao ano } 2000 \text {. A CPUE variou } \\
\text { globalmente entre } 0 \text { e } 14 \text { e } 0 \text { e } 2,4 \text { para } \\
\text { CC e DC, respectivamente. Estimou-se } \\
\text { uma captura entre } 150 \text { e } 200 \text { mil CC e } 30 \\
\text { a } 60 \text { mil DC pata todo o Atlântico. }\end{array}$ \\
\hline $\begin{array}{l}\text { Pinedo \& Polachek } \\
\text { (2004) }\end{array}$ & $\mathrm{CC} / \mathrm{DC} / \mathrm{LO}$ & $\begin{array}{l}\text { Espinhel } \\
\text { pelágico }\end{array}$ & - & OC & $\begin{array}{l}\text { Dados de quatro cruzeiros de pesquisa } \\
\text { realizados nos anos de } 1996 \text { e } 1999 \text { ao } \\
\text { largo do sul do Brasil. Foram capturadas } \\
19 \text { tartarugas marinhas, com uma CPUE } \\
\text { de 1,5, incluindo CC (CRC entre } 51 \text { e } 67 \\
\mathrm{~cm} \text { ), LO (CRC de } 47,1 \text { e } 58,2 \mathrm{~cm} \text { ) e DC. }\end{array}$ \\
\hline
\end{tabular}


(continuação Tabela I)

\begin{tabular}{llcccc}
\hline Referência $^{2}$ & Espécie & $\begin{array}{c}\text { Pescaria } \\
\text { industrial }\end{array}$ & $\begin{array}{c}\text { Pescaria } \\
\text { artesanal }\end{array}$ & $\begin{array}{c}\text { Estado/ } \\
\text { região }\end{array}$ & Dados de captura \\
\hline
\end{tabular}

Dados de programas de observadores de bordo da frota pesqueira comercial do Brasil e Uruguai e de cruzeiros de pesquisa, correspondentes ao período

Domingo et al. (2006)

$\mathrm{CC} / \mathrm{DC} / \mathrm{LO} / \quad$ Espinhel $\quad$

OC de 1998 a 2004, ao largo da costa brasileira. Foram capturadas 1.128 CC (CPUE entre 0,1 e 1,3), 259 DC (CPUE entre 0,01 e 0,2$), 28 \mathrm{CM}$ (CPUE inferior a 0,02), 29 LO (CPUE inferior a 0,02) e 42 tartarugas não identificadas.

Dados de monitoramento de pescarias

Cerco-

flutuante; Rede de emalhe; Rede de tróia; Arrasto de fundo;
Espinhel pelágico; Rede de

Deriva.
Diversas

$\mathrm{CM} / \mathrm{CC} / \mathrm{E}$

Marcovaldi et al. (2006)

$\mathrm{DC} / \mathrm{LO}$ e reportados por pescadores entre 1991 e 1998. Foram registradas captu-

SP ras de $2.475 \mathrm{CM}$ (CCC entre 27 e 96 $\mathrm{cm}, 81,1 \%$ em cerco flutuante), $23 \mathrm{EI}$ (CCC entre 32 e $67 \mathrm{~cm}$ ), e 13 CC (CCC entre 32,5 e $85 \mathrm{~cm}$ ).

Dados de monitoramento do Projeto TAMAR-ICMBio de pescarias costeiras desde 1990 e oceânicas desde 2001. Foram identificadas 16 pescarias costeiras e duas oceânicas que interagiram com as cinco espécies de tartarugas marinhas do Brasil. Não apresentam dados de captura

Dados obtidos por entrevistas com pescadores em 2003 na Ilha de Santa Catarina. As redes de emalhe com maior probabilidade de captura foram a feiticeira (reportada por $45 \%$ dos pescadores), espera de superfície ou fundo (39\% dos pescadores), redes de malha grande (21\% dos pescadores), redes de fundo de espera ou caceio para peixes ( $12 \%$ dos pescadores) e redes fixas de cerco flutuante $(12 \%)$. CM e CC foram as principais espécies mencionadas.

Relato de uma DC encalhada vindo a óbito na praia de Mundaú - CE, por interação com espinhel pelágico.

Dados de programa de observadores de bordo da frota pesqueira comercial do Brasil e Uruguai e de cruzeiros de pesquisa correspondentes ao período de 1998 a 2005, ao largo da costa brasileira. Foram capturadas 1.693 CC (CPUE entre 0,42 e 2,45; CCC entre 38 e $83 \mathrm{~cm}$ ) e 238 DC (CPUE entre 0,05 e 0,7; CCC entre 115 e $194 \mathrm{~cm}$ ).

Dados de cruzeiros de pesquisa correspondentes ao período de 2001 a 2006, ao largo da costa brasileira, de literatura e de entrevistas com mestres de embarcações e tripulação. Foram capturadas 45 tartarugas marinhas nas pescar-

Pargueira;

Vara e iscaviva;

Espinhel

pelágico.
OC ias de espinhel pelágico, incluindo $\mathrm{CC}$ (CPUE médio de 0,52; CCC entre 64 a $80 \mathrm{~cm}$ ), DC (CPUE médio de 0,34; CCC de $131 \mathrm{~cm}), \mathrm{CM}$ (CCC entre 36 e $52 \mathrm{~cm}$ ) e LO (CCC de $59 \mathrm{~cm}$ ). Não foram registradas capturas nas pescarias de corri$\mathrm{co}$, pargueira e vara e isca-viva. 
(continuação Tabela l)

\begin{tabular}{|c|c|c|c|c|c|}
\hline Referência ${ }^{2}$ & Espécie & $\begin{array}{c}\text { Pescaria } \\
\text { industrial }\end{array}$ & $\begin{array}{c}\text { Pescaria } \\
\text { artesanal }\end{array}$ & $\begin{array}{c}\text { Estado/ } \\
\text { região }\end{array}$ & Dados de captura \\
\hline Giffoni et al. (2008) & $\mathrm{CC} / \mathrm{DC}$ & $\begin{array}{l}\text { Espinhel } \\
\text { pelágico }\end{array}$ & & OC & $\begin{array}{l}\text { Dados de programa de observadores } \\
\text { de bordo da frota pesqueira comercial } \\
\text { do Brasil e Uruguai e de cruzeiros de } \\
\text { pesquisa correspondentes ao período } \\
\text { de } 2005 \text { a } 2007 \text {, ao largo da costa } \\
\text { brasileira. Foram capturadas } 1.965 \text { CC } \\
\text { (CPUE média de } 0,53 \text {; CCC entre } 32 \text { e } \\
109 \mathrm{~cm} \text { ) e } 312 \text { DC (CPUE média de } \\
0,08 \text {; CCC entre } 80 \text { e } 170 \mathrm{~cm} \text { ). }\end{array}$ \\
\hline
\end{tabular}

Nagoaka et al. (2008) CM $\quad-\quad$ Cerco-fixo $\quad$ SP

Dados obtidos do monitoramento de cercos-fixos entre 2004 e 2007. Foram capturadas 163 CM (CCC médio de $37,7 \mathrm{~cm}$ ).

Dados de observadores de bordo da frota pesqueira comercial brasileira e estrangeira correspondentes ao período de 2001 a 2005, ao largo da costa brasileira. Foram capturadas 789 CC

Sales et al. (2008)

$\mathrm{CC} / \mathrm{DC} /$

Espinhel

$\mathrm{CM} / \mathrm{LO}$ pelágico

OC (maior CPUE registrado de 2,17; CCC entre 39 e $103 \mathrm{~cm}$ ), 341 DC (maior CPUE registrado de 0,98 ; CCC entre 50 e $194 \mathrm{~cm}$ ), 45 CM (maior CPUE registrado de 0.15; CCC entre 27 e $90 \mathrm{~cm}$ ), e 81 LO (maior CPUE registrado de 0,01 ; CCC entre 35 e $80 \mathrm{~cm}$ ).

Dados de observadores de bordo da frota pesqueira comercial uruguaia cor-

Pons et al. (2009)

CC $\quad \begin{aligned} & \text { Espinhel } \\ & \text { pelágico }\end{aligned}$

OC respondentes ao período de 1998 a 2007, ao largo do sudoeste do Atlântico. Foram capturadas $2.261 \mathrm{CC}$ (CPUE entre 0,4 e 2,0$)$.

\begin{tabular}{lll}
\hline & & $\begin{array}{l}\text { Arrasto de } \\
\text { fundo; Linha } \\
\text { de mão; } \\
\text { Soares et al. (2009) }\end{array}$ \\
& $\mathrm{CM} / \mathrm{CC} / \mathrm{EI} / \mathrm{LO}$ & Arrasto de \\
& praia \\
\hline
\end{tabular}

Bahia \& Bondioli (2010)

$\mathrm{CM} / \mathrm{CC} / \mathrm{EI}$

- $\quad$ Cerco-fixo

SP

Dados obtidos através de entrevistas com pescadores artesanais em 2009. $\mathrm{CM}$ foi a espécie mais capturada (reportada por 67,5\% dos pescadores).

Dados obtidos por entrevistas com pescadores da região de Cananéia-SP entre 2007 e 2008. A maior parte dos pescadores $(55 \%)$ reportaram a captura de tartarugas marinhas nos cercos-fixos, das espécies CM, CC e EI.

Dados obtidos através de entrevistas com mestres de embarcação e de observadores de bordo entre 2004 a

Lima et al. (2010)

$\mathrm{CM} / \mathrm{CC} / \mathrm{EI}$

Rede de emalhe (caçoeira)

CE 2006. Foram registradas a captura de 61 EI (CCC entre 35,7 e 43,2 cm), 54 CM (CCC entre 34,3 e $39,7 \mathrm{~cm}$ ) e 6 CC em redes de emalhe (caçoeira). A CPUE (tartarugas capturadas por barco por ano) variou entre 0,43 e 1,0. Dados de observadores de bordo da frota pesqueira comercial do Brasil e

Pons et al. (2010)

$\mathrm{CC}$

Espinhel
pelágico
OC
Uruguai correspondentes ao período de 1998 a 2007, ao largo do sudoeste do Atlântico. Foram capturadas 3.778 CC (CPUE entre 0,38 e 1,78 ). 
(continuação Tabela I)

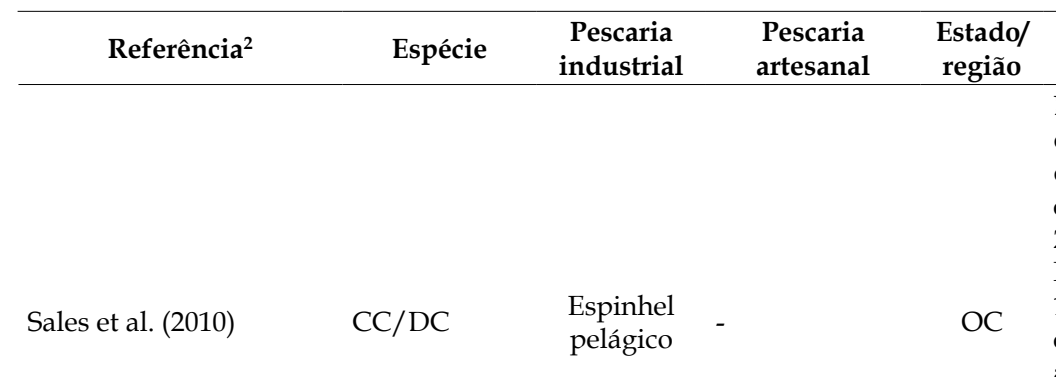

Dados de programa de observadores de bordo da frota pesqueira comercial do Brasil e de cruzeiros de pesquisa correspondentes ao período de 2004 a 2008, ao largo da costa brasileira. Foram capturadas 170 CC (CPUE de 1,61 no anzol J e de 0,73 no anzol circular; CCC entre 50 e $81 \mathrm{~cm}$ no anzol J e entre 37,5 e $96 \mathrm{~cm}$ no anzol circular), 28 DC (CPUE de 0,27 no anzol J e de 0,10 no anzol circular; CCC entre 127 e $149 \mathrm{~cm})$ e $2 \mathrm{CM}$ (CPUE de 0,01 no anzol J e de 0,01 no anzol circular).

Dados obtidos de cruzeiros com embarcações comerciais entre 2006

Pacheco et al. (2011) DC/CM/LO Espinhel - OC e 2007, ao largo da costa NE do Brasil. Foram capturadas 19 DC, 10 CM e 1 LO;

Dados obtidos através de embarques na frota artesanal estuarina e costeira e por entrevistas com pescadores no período de 2007 e 2008. Foram capturadas 49

López-Barrera et al (2012)

$\mathrm{CM}$

Rede de emalhe

PR

$\mathrm{CM}$ (CCC entre 25 e $55 \mathrm{~cm}$ ) em redes de emalhe (fixas, caceio e cerco), com uma taxa de mortalidade de $63 \%$, correlacionadas com o tempo de imersão das redes e o tamanho das malhas.

Dados obtidos por mestres de embarcações da frota comercial sediada em Ubatuba-SP e Itajaí-SC no período de 2002 a 2008, na plataforma continental

Rede de deriva do sul-sudeste do Brasil. Foram capturadas $252 \mathrm{DC}$ (CPUE de 0,14 tartarugas por $\mathrm{km}$ de rede), $47 \mathrm{CC}$ (CPUE de 0,03 tartarugas por $\mathrm{km}$ de rede), $27 \mathrm{CM}$ (CPUE de 0,02 tartarugas por $\mathrm{km}$ de rede) e 25 de espécie não-identificada.

Revisão bibliográfica de trabalhos com

$\begin{array}{lll}\text { Coelho et al. (2013) } & \begin{array}{l}\mathrm{CM} / \mathrm{CC} / \\ \mathrm{DC} / \mathrm{LO}\end{array} & \begin{array}{l}\text { Espinhel } \\ \text { pelágico }\end{array}\end{array} \quad$ OC

de CPUE das seis espécies de tartaruga ao redor do mundo entre 2006 a 2013.

Foram realizadas entrevistas com 20 pescadores de embarcações de médio porte e 34 pescadores de barcos de grande escala (selecionados aleatoriamente). As entrevistas foram realizaRamos e Vasconcelhos (2013)

$\mathrm{CM} / \mathrm{CC} / \mathrm{DC} \quad$ - $\quad$ Rede de emalhe
das entre setembro de 2010 e outubro de 2011. Dos 54 entrevistados, 50 $(92,6 \%)$ relataram a captura incidental de uma ou mais tartarugas a cada ano. As espécies mais capturadas e avistadas no RS foram CM, CC e DC.

Dados de programa de observadores de bordo da frota pesqueira comercial do Brasil e Uruguai correspondentes ao período de 1998 a 2010, ao largo da costa brasileira. Foram capturadas 8.573 CC (CPUE média de 0,53; CCC entre 32 e $109 \mathrm{~cm}$ ), 1.379 DC (CPUE média de 0,08; CCC entre 80 e $170 \mathrm{~cm}$ ) e 600 LO. 
(continuação Tabela I)

\begin{tabular}{|c|c|c|c|c|c|}
\hline Referência $^{2}$ & Espécie & $\begin{array}{c}\text { Pescaria } \\
\text { industrial }\end{array}$ & $\begin{array}{c}\text { Pescaria } \\
\text { artesanal }\end{array}$ & $\begin{array}{c}\text { Estado/ } \\
\text { região }\end{array}$ & Dados de captura \\
\hline $\begin{array}{l}\text { Braga \& Schiavetti } \\
\text { (2015) }\end{array}$ & $\begin{array}{l}\mathrm{EI} / \mathrm{CM} / \mathrm{CC} / \\
\mathrm{DC}\end{array}$ & - & $\begin{array}{l}\text { Linha-demão; } \\
\text { rede de emalhe; } \\
\text { arrasto de } \\
\text { fundo. }\end{array}$ & BA & $\begin{array}{l}\text { Dados obtidos por entrevistas com } \\
\text { pescadores da região de Ilhéus-BA en- } \\
\text { tre } 2010 \text { e } 2011 \text {. Os pescadores repor- } \\
\text { taram capturar tartarugas marinhas } \\
\text { nas pescarias de linha-de-mão e redes } \\
\text { de emalhe e redes de arrasto. }\end{array}$ \\
\hline Brito et al. (2015) & $\begin{array}{l}\text { CM/DC/EI/ } \\
\text { CC/LO }\end{array}$ & - & $\begin{array}{l}\text { Rede de } \\
\text { emalhe; } \\
\text { espinhel; cerco- } \\
\text { fixo (curral) }\end{array}$ & PA & $\begin{array}{l}\text { Dados obtidos por entrevistas com } \\
\text { pescadores da região de São João de } \\
\text { Pirabas-PA entre } 2013 \text { e } 2014 \text { C. Captu- } \\
\text { ras incidentais foram relatadas por } \\
76 \% \text { dos pescadores, ocorrendo princi- } \\
\text { palmente em redes, espinheis e currais. }\end{array}$ \\
\hline Monteiro et al. (2016) & $\mathrm{CM} / \mathrm{CC} / \mathrm{DC}$ & $\begin{array}{l}\text { Espinhel } \\
\text { pelágico; } \\
\text { Rede de } \\
\text { emalhe de } \\
\text { fundo; } \\
\text { Arrasto de } \\
\text { parelha; } \\
\text { Arrasto } \\
\text { duplo }\end{array}$ & - & RS & $\begin{array}{l}\text { Dados obtidos a partir do } \\
\text { monitoramento de encalhes de praia } \\
\text { na costa do Rio Grande do Sul entre } \\
1995 \text { e 2014, avaliando sinais visíveis } \\
\text { de interação com a pesca. Foram } \\
\text { constados sinais de interação em 1,2\% } \\
\text { dos encalhes, relacionados ao espinhel } \\
\text { pelágico, às redes de emalhe de fundo, } \\
\text { arrasto-duplo e arrasto de parelha. As } \\
\text { espécies observadas com sinais de } \\
\text { interação foram CC, CM e DC. }\end{array}$ \\
\hline Nogueira \& Alves (2016) & $\mathrm{CM} / \mathrm{CC} / \mathrm{EI}$ & - & $\begin{array}{l}\text { Rede de } \\
\text { emalhe; linha } \\
\text { de mão; arrasto } \\
\text { de fundo. }\end{array}$ & PB & $\begin{array}{l}\text { Dados obtidos por entrevistas com } 67 \\
\text { pescadores da região costeira da } \\
\text { Paraíba-PB, entre fevereiro e agosto de } \\
2014 \text {. Capturas incidentais foram } \\
\text { relatadas por } 31,6 \% \text { dos pescadores de } \\
\text { linha de mão, } 60,4 \% \text { com rede de } \\
\text { emalhe. As espécies relatadas foram } \\
\text { CC, CM e EI. }\end{array}$ \\
\hline
\end{tabular}

${ }^{1}$ Dados de CPUE para o Espinhel pelágico se refere ao número de tartarugas capturadas por 1.000 anzóis. As demais formas de cálculo da CPUE estão indicadas quando mencionadas.

${ }^{2}$ As referências encontram-se no Apêndice 1. 
Tabela II. Medidas mitigatórias e planos/ações de conservação propostos de acordo com as pescarias, as espécies de tartarugas marinhas envolvidas e outras espécies citadas. $\mathrm{DC}=$ Dermochelys coriacea; $\mathrm{CC}=$ Caretta caretta; $\mathrm{LO}=$ Lepidochelys olivacea; $\mathrm{CM}=$ Chelonia mydas; $\mathrm{EI}=$ Eretmochelys imbricata.

\begin{tabular}{|c|c|c|c|c|c|}
\hline Referência $^{1}$ & $\begin{array}{l}\text { Espécies } \\
\text { citadas }\end{array}$ & $\begin{array}{l}\text { Pescaria } \\
\text { industrial }\end{array}$ & $\begin{array}{l}\text { Pescaria } \\
\text { artesanal }\end{array}$ & $\begin{array}{l}\text { Outras } \\
\text { espécies } \\
\text { citadas }\end{array}$ & $\begin{array}{l}\text { Medidas mitigatórias e planos/ações de } \\
\text { conservação propostos }\end{array}$ \\
\hline $\begin{array}{l}\text { Marcovaldi et al. } \\
(2002 a)\end{array}$ & $\begin{array}{l}\mathrm{CM} / \mathrm{CC} / \\
\mathrm{EI} / \mathrm{DC} / \mathrm{LO}\end{array}$ & $\begin{array}{l}\text { Espinhel } \\
\text { pelágico }\end{array}$ & - & $\begin{array}{l}\text { Aves } \\
\text { marinhas }\end{array}$ & $\begin{array}{l}\text { Implementação do Plano de Ação do Projeto } \\
\text { TAMAR/ICMBio para Redução de Capturas } \\
\text { Incidentais de Tartarugas Marinhas pela } \\
\text { Atividade Pesqueira. }\end{array}$ \\
\hline $\begin{array}{l}\text { Marcovaldi et al. } \\
(2002 b)\end{array}$ & $\begin{array}{l}\mathrm{CM} / \mathrm{CC} / \\
\mathrm{EI} / \mathrm{DC} / \mathrm{LO}\end{array}$ & $\begin{array}{l}\text { Espinhel } \\
\text { pelágico }\end{array}$ & - & $\begin{array}{l}\text { Aves } \\
\text { marinhas }\end{array}$ & $\begin{array}{l}\text { Implementação do Plano de Ação do Projeto } \\
\text { TAMAR/ICMBio para Redução de Capturas } \\
\text { Incidentais de Tartarugas Marinhas pela } \\
\text { Atividade Pesqueira. }\end{array}$ \\
\hline Kotas et al. (2004) & $\mathrm{CC} / \mathrm{DC}$ & $\begin{array}{l}\text { Espinhel } \\
\text { pelágico }\end{array}$ & - & - & $\begin{array}{l}\text { Propõe a implementação de um Programa } \\
\text { Internacional de Observação de Bordo de } \\
\text { Espinheleiros no Atlântico Sul. }\end{array}$ \\
\hline $\begin{array}{l}\text { Domingo et al. } \\
(2006)\end{array}$ & $\begin{array}{l}\mathrm{CM} / \mathrm{CC} / \\
\mathrm{DC} / \mathrm{LO}\end{array}$ & $\begin{array}{l}\text { Espinhel } \\
\text { pelágico }\end{array}$ & - & - & $\begin{array}{l}\text { Como uma estratégia de conservação, a partir } \\
\text { da verificação de áreas de maior concentração } \\
\text { das tartarugas, aprofundar os estudos ambien- } \\
\text { tais nestas regiões. }\end{array}$ \\
\hline $\begin{array}{l}\text { Marcovaldi et al. } \\
(2006)\end{array}$ & $\begin{array}{l}\mathrm{CM} / \mathrm{CC} / \\
\mathrm{EI} / \mathrm{DC} / \mathrm{LO}\end{array}$ & $\begin{array}{l}\text { Espinhel } \\
\text { pelágico; } \\
\text { Rede de } \\
\text { deriva }\end{array}$ & Diversas & - & $\begin{array}{l}\text { Propõe, dentro de um plano estratégico para } \\
\text { conservação, caracterizar a pesca (CPUE, região, } \\
\text { época), identificar as ameaças as espécies de } \\
\text { tartarugas marinhas durante suas fases de vida, } \\
\text { melhorar a gestão da pesca e realizar campanhas } \\
\text { de sensibilização para os pescadores. }\end{array}$ \\
\hline Sales et al. (2006) & $\mathrm{CC}$ & $\begin{array}{l}\text { Espinhel } \\
\text { pelágico }\end{array}$ & & $\begin{array}{l}\text { Aves } \\
\text { marinhas }\end{array}$ & $\begin{array}{l}\text { Realizar testes com redes de emalhe na cor azul e } \\
\text { com óleo/resina com sabor desagradável, como } \\
\text { alternativas para diminuir a captura incidental. }\end{array}$ \\
\hline $\begin{array}{l}\text { Nagoaka et al. } \\
(2008)\end{array}$ & $\mathrm{CM}$ & - & Cerco-fixo & - & $\begin{array}{l}\text { Propõe o monitoramento das áreas com maior } \\
\text { incidência de tartarugas e catalogar as regiões } \\
\text { mais críticas à captura pela pesca. }\end{array}$ \\
\hline $\begin{array}{l}\text { Damasio e } \\
\text { Carvalho (2010) }\end{array}$ & $\begin{array}{l}\mathrm{CM} / \mathrm{CC} / \\
\mathrm{EI} / \mathrm{DC} / \mathrm{LO}\end{array}$ & - & $\begin{array}{l}\text { Rede de } \\
\text { emalhe; } \\
\text { Cerco-fixo; } \\
\text { Linha de mão }\end{array}$ & - & $\begin{array}{l}\text { Recomenda a implementação de programa de } \\
\text { gestão para tartarugas marinhas nas comuni- } \\
\text { dades onde há ocorrência de tartarugas, envol- } \\
\text { vendo os membros nas atividades e destacando: } \\
\text { a) informação científica - transmitir conheci- } \\
\text { mentos aos pescadores sobre a situação das es- } \\
\text { pécies ameaçadas; b) programa de incentivo } \\
\text { para registrar a captura e fazer a marcação das } \\
\text { tartarugas capturadas incidentalmente; e c) for- } \\
\text { mação e capacitação de membros da comuni- } \\
\text { dade para monitorar e marcar as tartarugas. }\end{array}$ \\
\hline Lima et al. (2010) & $\mathrm{CM} / \mathrm{CC} / \mathrm{EI}$ & - & $\begin{array}{l}\text { Rede de } \\
\text { emalhe }\end{array}$ & - & $\begin{array}{l}\text { Propõe algumas ações que podem favorecer o } \\
\text { cumprimento da legislação por parte dos pesca- } \\
\text { dores e reduzir o impacto da pesca da lagosta } \\
\text { sobre as tartarugas, sendo: a) maior fiscalização } \\
\text { do poder público; b) incentivos ao uso de ar- } \\
\text { madilhas para a captura da lagosta, por exemplo, } \\
\text { através de oficinas de capacitação na construção } \\
\text { de armadilhas; c) o desenvolvimento de alternati- } \\
\text { vas de pesca aos pescadores, o que poderia re- } \\
\text { duzir o número de embarcações voltadas à pesca } \\
\text { da lagosta; d) o estabelecimento de áreas de ex- } \\
\text { clusão de pesca da lagosta; e e) atividades de edu- } \\
\text { cação e sensibilização aos pescadores. }\end{array}$ \\
\hline Sales (2010) & $\mathrm{CC} / \mathrm{DC}$ & $\begin{array}{l}\text { Espinhel } \\
\text { pelágico }\end{array}$ & - & $\begin{array}{c}\text { Aves } \\
\text { marinhas }\end{array}$ & $\begin{array}{l}\text { Propõe testar a eficácia do uso de anzol circular } \\
\text { em substituição ao anzol tipo J. }\end{array}$ \\
\hline Silva et al. (2010) & $\mathrm{LO}$ & - & $\begin{array}{l}\text { Arrasto de } \\
\text { fundo }\end{array}$ & - & $\begin{array}{l}\text { Duas estratégias desenvolvidas pelo projeto } \\
\text { TAMAR/ICMBio: a) implementação de um } \\
\text { programa de monitoramento marinho; e b) } \\
\text { participação ativa em fóruns locais. }\end{array}$ \\
\hline $\begin{array}{l}\text { Pacheco et al. } \\
(2011)\end{array}$ & $\begin{array}{l}\mathrm{CM} / \mathrm{CC} / \\
\mathrm{EI} / \mathrm{DC} / \mathrm{LO}\end{array}$ & $\begin{array}{l}\text { Espinhel } \\
\text { pelágico }\end{array}$ & - & $\begin{array}{c}\text { Aves } \\
\text { marinhas }\end{array}$ & Testar o uso do anzol circular. \\
\hline
\end{tabular}


(continuação Tabela II)

\begin{tabular}{cccccc}
\hline Referência & $\begin{array}{c}\text { Espécies } \\
\text { citadas }\end{array}$ & $\begin{array}{c}\text { Pescaria } \\
\text { industrial }\end{array}$ & $\begin{array}{c}\text { Pescaria } \\
\text { artesanal }\end{array}$ & $\begin{array}{c}\text { Outras } \\
\text { espécies } \\
\text { citadas }\end{array}$ & $\begin{array}{c}\text { Medidas mitigatórias e planos/ações de } \\
\text { conservação propostos }\end{array}$ \\
\hline
\end{tabular}

\begin{tabular}{|c|c|c|c|c|c|}
\hline $\begin{array}{l}\text { Santos et al. } \\
(2011)\end{array}$ & $\mathrm{CC}$ & $\begin{array}{l}\text { Espinhel } \\
\text { pelágico }\end{array}$ & $\begin{array}{l}\text { Rede de } \\
\text { emalhe de } \\
\text { fundo; Rede } \\
\text { de emalhe de } \\
\text { superfície; } \\
\text { Arrasto de } \\
\text { fundo; Cerco } \\
\text { flutuante }\end{array}$ & - & $\begin{array}{l}\text { Propõe a continuidade da sensibilização pública } \\
\text { e o desenvolvimento local através das atividades } \\
\text { de educação ambiental e turismo, a manutenção } \\
\text { de monitoramento nas áreas de desova, e o } \\
\text { aumento das pesquisas de longo prazo. }\end{array}$ \\
\hline $\begin{array}{l}\text { Fiedler et al. } \\
(2012)\end{array}$ & $\begin{array}{l}\mathrm{CM} / \mathrm{CC} / \\
\mathrm{DC}\end{array}$ & - & $\begin{array}{l}\text { Rede de } \\
\text { deriva }\end{array}$ & - & $\begin{array}{l}\text { Recomenda a proibição deste tipo de pesca na } \\
\text { região. }\end{array}$ \\
\hline
\end{tabular}
Necessidade de um plano de manejo para redução de capturas nas áreas de alimentação, que considere, para a região, ao invés da criação de áreas de exclusão de pesca ou de restrições às pescarias. Sugere também, o desenvolvimento de medidas regulatórias sobre as técnicas de pesca, tais como o período de imersão, o comal. (2012) emalhe
López-Barrera et Rede de
$\mathrm{CM}$ primento ou o uso sazonal das redes. Paras as áreas costeiras, sugere a colocação das redes em paralelo ao invés de perpendicular, como usual. $\mathrm{O}$ teste deste método deve ser feito conjuntamente com os pescadores. No ambiente estuarino, deve-se identificar área de maior ocorrência de tartarugas e adequar as práticas de pesca nestes locais. Além disso, o desenvolvimento de atividades de educação ambiental para a população local e aos pescadores.

Silva et al. (2012) CM/CC/ $\quad$ - _ n $\quad$ - $\quad$ Propõe a criação de centros de reabilitação
$\mathrm{EI} / \mathrm{DC} / \mathrm{LO}$ - $\quad$ - $\quad$ - $\quad$ associados a educação ambiental local.

Propõe caracterizar os comportamentos das tartarugas marinhas e identificar as áreas mais utilizadas, uma vez que o uso varia no tempo e no espaço, sendo importante para o planejamento da conservação. Em parceria com o Uruguai, 27 transmissores satelitais foram implantados em CC capturada incidentalmente em espinhel pelágico uruguaio e brasileiro que operam no Oceano Atlântico Sudoeste.

Recomenda o trabalho conjunto com pescadores (que possam testar equipamentos de pesca), criação de medidas práticas pra diminuir a captura, envolver a comunidade na gestão costeira das áreas protegidas, garantir o cumprimento das leis.

$\mathrm{EI} / \mathrm{DC}$

Rede de

emalhe

Propõe projetos de extensão para sensibilizar os

Rede de

emalhe; Linha

de mão;

Brito et al. (2015)

$\mathrm{CM} / \mathrm{CC} /$ $\mathrm{EI} / \mathrm{DC} / \mathrm{LO}$ Espinhel

Cerco-fixo;

Tarrafa;

Tapagem²; Matapi $^{3}$ pescadores a respeito das ameaças que as populações de tartarugas marinhas têm sofrido decorrentes da atividade de pesca e outras ações antropogênicas, bem como o monitoramento das capturas incidentais.

\begin{tabular}{|c|c|c|}
\hline $\begin{array}{l}\text { Monteiro et al. } \\
\text { (2016) }\end{array}$ & $\begin{array}{l}\mathrm{CM} / \mathrm{CC} / \\
\mathrm{DC}\end{array}$ & $\begin{array}{l}\text { Espinhel } \\
\text { pelágico; } \\
\text { Rede de } \\
\text { emalhe de } \\
\text { fundo; } \\
\text { Arrasto de } \\
\text { parelha; } \\
\text { Arrasto } \\
\text { duplo }\end{array}$ \\
\hline
\end{tabular}




\begin{tabular}{|c|c|c|c|c|c|}
\hline Referência $^{1}$ & $\begin{array}{l}\text { Espécies } \\
\text { citadas }\end{array}$ & $\begin{array}{l}\text { Pescaria } \\
\text { industrial }\end{array}$ & $\begin{array}{l}\text { Pescaria } \\
\text { artesanal }\end{array}$ & $\begin{array}{l}\text { Outras } \\
\text { espécies } \\
\text { citadas }\end{array}$ & $\begin{array}{c}\text { Medidas mitigatórias e planos/ações de } \\
\text { conservação propostos }\end{array}$ \\
\hline
\end{tabular}

Recomendam: a) menor intervalo de tempo entre as vistorias dos pescadores às redes de emalhe; b) instrução aos pescadores sobre o manejo de animais capturados, evitando danos como a permanência de linhas nos animais; c)

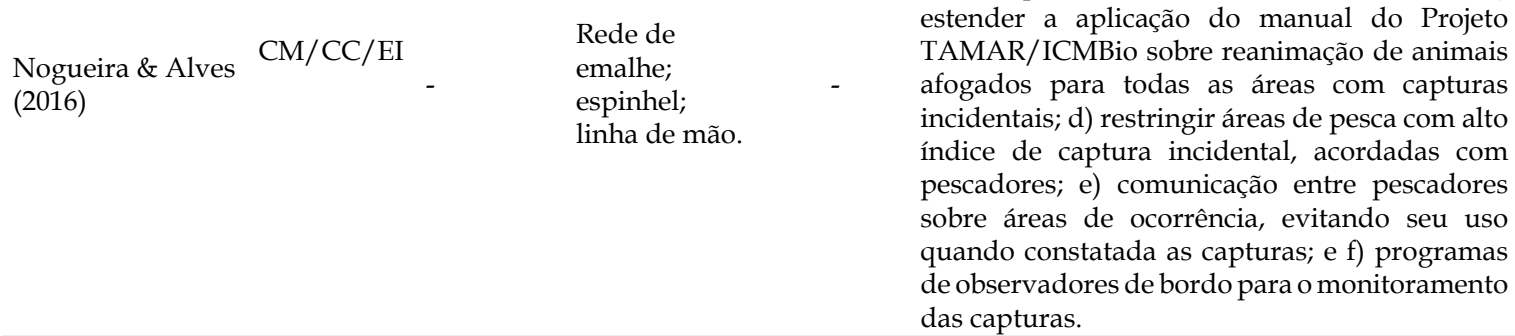

${ }^{1}$ As referências encontram-se no Apêndice 1.

${ }^{2}$ Pesca de tapagem - Pode ser feita com linha de nylon ou com o pari, uma espécie de esteira feita de talas de marajá (Pyrenoglyphis maruja). A técnica consiste em cruzar um igarapé com estas linhas ou com as esteiras, fixando-as a varas cravadas (paritás) no chão, impedindo a fuga dos peixes (tapagem do igarapé).

${ }^{3}$ Pesca com matapi: armadilha de forma cilíndrica, fechada por dois cones por onde entram os peixes e ficam presos. Assim como o cacuri, o matapi é confeccionado com talas de jupati (Raphia taedigera), porém este é mais alongado.

\section{DISCUSSÃO}

O aumento das publicações sobre interação de tartarugas marinhas e pesca está diretamente relacionado com a criação do Programa Nacional de Conservação das Tartarugas Marinhas (Projeto TAMAR-ICMBio), em 1980. Inicialmente com o objetivo de identificar as principais áreas de reprodução das cinco espécies de tartarugas marinhas que ocorrem no Brasil e as ameaças à sobrevivência destas populações (Marcovaldi \& Marcovaldi, 1999), a partir de 1991, os esforços se ampliaram também para as áreas de alimentação (Marcovaldi et al., 1998). A partir dos anos 2000, o TAMAR-ICMBio desenvolveu um plano específico para lidar com a problemática da interação com a pesca (Marcovaldi et al., 2002). Sendo assim, o aumento significativo de publicações ocorre a partir deste período, quando o tema ganhou maior notoriedade e o assunto passou a ser discutido em diferentes instâncias, desde fóruns locais até intergovernamentais (Marcovaldi et al., 2002). Além do TAMARICMBio, outras instituições de pesquisa e conservação também se dedicam a estudar esta temática, contribuindo para o aumento das publicações no período. A maioria dos trabalhos publicados estão em língua inglesa e em periódicos internacionais, o que vai de encontro à tendência mundial de difusão do conhecimento científico, além de permitir o maior compartilhamento de conhecimento sobre conservação de tartarugas marinhas, uma vez que estas ações por vezes dependem de colaboração entre países (Hamann et al., 2010).

A maior parte dos trabalhos publicados abrangeram as águas oceânicas, isto porque os dados para as pesquisas são coletados por observadores a bordo de navios espinheleiros. O Decreto 4.810 de 19 de agosto de 2003 torna obrigatória a presença de um observador de bordo em embarcações estrangeiras, resultando em um maior esforço de monitoramento e documentação das capturas de tartarugas. Além do acompanhamento das pescarias e da geração de dados de captura, a convivência e interação cotidiana entre os observadores e a tripulação potencialmente promove mudanças nas concepções dos pescadores em relação à conservação das espécies (Galvão et al., 2010). 
O segundo maior número de publicações está no estado de São Paulo, o que pode ser atribuído a três fatores: uma das bases mais antigas do TAMAR-ICMBio em área de alimentação de tartarugas marinhas e com ocorrência de captura incidental (Gallo et al., 2006); a existência de diversas instituições de pesquisa/extensão e universidades; e o fato do sul e sudeste sediarem importantes frotas industriais, o que facilita o registro das informações sobre interação. As regiões norte e nordeste são importantes locais de reprodução e alimentação de quase todas as espécies que ocorrem no Brasil (Marcovaldi \& Marcovaldi, 1999) e, ao mesmo tempo, apresentam pescarias artesanais bastante diversificadas (Marcovaldi et al., 2006), bem como importantes frotas industriais (Sales et al., 2008). Porém, aparentemente, ainda há um baixo esforço de pesquisa e/ou publicação dos resultados.

Observou-se que as espécies com mais dados de captura foram C. caretta, D. coriacea e C. mydas. Isto se explica em parte pela abundância e distribuição das espécies (Almeida et al., 2011a; 2011b; Santos et al., 2011), bem como pela interação com diferentes pescarias, tanto artesanais quanto industriais. C. caretta e D. coriacea estão presentes em águas oceânicas, a primeira principalmente em estágio juvenil e a segunda tanto jovem quanto adulta, e são as espécies que mais interagem com a pesca de espinhel (Sales et al., 2008). Isto explica a maior ocorrência destas espécies nos estudos sobre interação com a pesca, uma vez que o espinhe-pelágico também é a pescaria com maior esforço de monitoramento. $C$. mydas pela sua grande abundância em águas costeiras em praticamente todo o litoral brasileiro, é a espécie que mais interage com a pesca artesanal, principalmente por ser praticada em águas costeiras.

Apesar de E. imbricata e L. olivacea estarem presentes em toda a costa brasileira, a primeira é mais abundante apenas na região tropical, tanto em áreas costeiras, quanto em ilhas oceânicas (Marcovaldi et al., 2011), e a segunda com maior presença nas regiões de reprodução no nordeste e em águas oceânicas (Castilhos et al. 2011). Estas distribuições mais restritas explicam, em parte, sua menor frequência nos estudos de interação com a pesca. A captura de E. imbricata ocorre principalmente em pescarias costeiras com redes de emalhe associadas a fundos rochosos e recifes costeiros. Capturas de L. olivacea são reportadas tanto em pescarias costeiras de arrasto de camarões na costa de Sergipe (Silva et al., 2010), quanto em águas oceânicas do sul, sudeste e nordeste (Sales et al., 2008).

O espinhel-pelágico foi a pescaria mais reportada pela interação com a pesca no Brasil. Além de ser uma pescaria presente ao largo da costa brasileira, há um esforço de monitoramento em nível mundial, o que inclui as espécies capturadas incidentalmente, como as tartarugas marinhas (Lewison \& Crowder, 2004). No Brasil, o embarque de um observador de bordo é obrigatório desde 2003, durante os cruzeiros de pesca estrangeiros. Em 2006, com a instrução normativa conjunta SEAP/MMA N 1, é instituído o Programa Nacional de Observadores de Bordo da Frota Pesqueira - PROBORDO, fortalecendo a obtenção de dados de capturas incidentais, porém este programa foi suspenso em 2013 sem expectativas de retorno.

Vem sendo sugerido dois padrões globais da interação com o espinhel pelágico (Lewison \& Crowder, 2004): uma proporção pequena das tartarugas capturadas morrem antes do recolhimento do espinhel, apesar de algumas morrerem ou não após o recolhimento; e sua captura é relativamente rara, o que explica valores de CPUE precedidos de muitos zeros. Porém, apesar do impacto individual de cada embarcação parecer pequeno, existem bilhões de anzóis de espinhel a cada ano, sugerindo um substancial impacto cumulativo (Lewison \& Crowder, 2004). No Brasil foram definidas quatro zonas de captura ao largo da costa brasileira (Sales et al., 2008): 1) a região norte; 2) a região central, incluindo 
as cadeias Vitória-Trindade e Abrolhos; 3) a região sul; e 4) representando as regiões de mar aberto - elevação do Rio Grande. As zonas 3 e 4 representam as áreas com maior índice de CPUE para C. caretta e D. coriacea. Apesar de ser a pescaria mais monitorada em termos de levantamento de CPUE no Brasil, a utilização das informações para a regulamentação das pescarias e de estudos sobre o cumprimento e avaliação das políticas, ainda são precárias, ameaçando as populações tanto das espécies-alvo quanto as capturadas incidentalmente (Fiedler et al., 2017).

As redes de emalhe, apesar de não contarem com o mesmo esforço de monitoramento e avaliação de mortalidade por pesca como o espinhel-pelágico, representam, em nível mundial, uma importante causa de redução das populações de tartarugas, talvez maior que o próprio espinhel, especialmente pelo alto índice de mortalidade durante a captura (Lewison \& Crowder, 2004). As características das redes no Brasil e das pescarias envolvidas (de artesanais a industriais) são muito variadas, com diferentes potenciais de captura, incluindo fatores espaciais e temporais (Marcovaldi et al., 2006). Fica evidente a ausência de dados de capturas e estimativas de CPUE para estas pescarias. Considerando que as águas costeiras são habitat para reprodução ou para alimentação de todas as espécies presentes na costa brasileira e, da mesma forma, importantes locais para diferentes pescarias de emalhe, especialmente da pesca artesanal, reforça-se a necessidade de estudos para melhor compreender as dinâmicas associadas a esta interação. Estudos de telemetria têm revelado corredores de migração e áreas de alimentação, com certo grau de fidelidade (Marcovaldi et al., 2010), para populações de fêmeas reprodutivas de C. caretta, E. imbricata e L. olivacea que se sobrepõem às áreas de pesca (Silva et al., 2011; Marcovaldi et al., 2010; Marcovaldi et al., 2012).

As próprias características multiespecíficas e diversidade de aspectos tecnológicos, socioeconômicos e culturais da pesca artesanal (Berkes et al., 2001; Diegues, 2004), dificultam o levantamento de dados sobre a interação com a pesca de maneira sistemática. Não há programas de monitoramento e os pescadores, em geral, tem receio de informar sobre suas atividades e as suas capturas incidentais (Biopesca, 2015). Métodos alternativos aos convencionais programas de monitoramentos e cruzeiros científicos tem sido propostos para o dimensionamento da interação com a pesca artesanal, tais como entrevistas com pescadores (Moore et al., 2010). Estudos de etnoconhecimento com pescadores sobre aspectos da bioecologia das tartarugas marinhas e de sua interação com a pesca vem trazendo informações relevantes para a compreensão desta relação em nível local, além de potencialmente fortalecer laços de confiança entre técnicos/pesquisadores e pescadores (Silva et al., 2010). O fomento de programas de monitoramento participativo da pesca (Malafaia et al., 2014; Dias et al., 2015), onde a dimensão da interação com as tartarugas marinhas poderia também ser considerada, vem ainda sendo pouco explorado no Brasil, mas seria uma alternativa para o estabelecimento de parcerias no levantamento de informações e criação de estratégias de mitigação das capturas (Bunce et al., 2000).

Quando consideradas as ações e medidas de mitigação propostas para reduzir ou lidar com a interação, poucas pescarias abrangem alterações tecnológicas. De fato, no caso do Brasil, há pouca literatura que descreva iniciativas de alterações tecnológicas, para além daquelas propostas para o espinhel pelágico. Para esta pescaria, a substituição dos tradicionais anzóis J pelo anzol circular vem sendo a principal medida testada no país (Sales et al., 2010), assim como ocorre ao redor do mundo. Para as demais pescarias, principalmente as redes de emalhe, alternativas tecnológicas são mais difíceis de serem desenvolvidas e implementadas (Samuel et al., 2005). Na pesca de arrasto, a despeito da existência da por- 
taria n. 5, de 19 de fevereiro de 1997 do IBAMA, que obriga a utilização de dispositivo de exclusão de tartarugas (Turtles Excluder Devices - TEDs) em embarcações a partir de $11 \mathrm{~m}$ de comprimento e/ou que utilizem mecanismos de recolhimento de redes a bordo, sua utilização ainda é baixa, assim como a fiscalização para garantir seu cumprimento (Gallo et al., 2006; Silva et al., 2010). Dependo das características do arrasto e de sua operação, por exemplo, arrastos realizados em períodos curtos, podem resultar na liberação de animais ainda vivos (Soto et al., 2002). Por outro lado, ainda é uma pescaria importante do ponto de vista da captura e mortalidade de tartarugas no país (Marcovaldi et al., 2006; Silva et al., 2010; Braga \& Schiavetti, 2013).

A maioria das ações de mitigação da interação de tartarugas com a pesca abrange ampliar o conhecimento sobre a interação, por exemplo, sobre as características dos petrechos, seu potencial em causar mortalidade e a identificação de áreas de maior ocorrência das capturas. Estas informações possibilitariam identificar áreas críticas de captura de tartarugas e propor mecanismos de exclusão de pesca nestas áreas e/ou alteração nos procedimentos de pesca (Nagoaka et al., 2008; Lima et al., 2010; Fiedler et al., 2012; LópezBarrera et al., 2012; Nogueira \& Alves, 2016; Monteiro et al., 2016). Para isto, o monitoramento vem sendo recomendado como imprescindível para a geração de informações. Dentre as ações mais frequentemente propostas incluem a sensibilização de pescadores sobre a problemática da captura de tartarugas e o desenvolvimento de programa de educação ambiental. Porém, a efetividade destas ações ainda carece de uma avaliação mais sistemática.

O envolvimento dos pescadores no processo de gestão se mostra uma alternativa para o seu maior engajamento nas estratégias de conservação (Silva et al. 2010). De fato, processos colaborativos de gestão da pesca e das zonas costeiras (e.g. cogestão) vem sendo propostos como uma alternativa aos modelos convencionais centralizadores de tomada de decisão (Armitage et al., 2009), com diversas iniciativas na costa brasileira (Kalikoski et al., 2009). Porém, a interação das tartarugas marinhas com a pesca enquanto elemento central do objetivo do arranjo de cogestão, ainda é incipiente na literatura internacional (Campbell et al., 2009) e nacional (Silva et al., 2010).

\section{CONCLUSÕES}

Com base nas três linhas norteadoras das pesquisas relacionadas à captura incidental de tartarugas marinhas pela pesca, visando avançar nas ações de conservação das espécies globalmente (Hamann et al., 2010), nesta revisão de literatura no Brasil, considerando a linha "avaliação das capturas e da mortalidade de tartarugas por área e pescarias", concluímos que:

(i) os esforços de avaliação das capturas e da mortalidade ainda está muito centrada na pesca industrial, em especial na pesca de espinhel-pelágico, na qual há avanços em determinar índices de CPUE e sua distribuição ao longo da costa;

(ii) os dados referentes à pesca artesanal são pontuais e escassos, sem monitoramento e avaliação sistemática das capturas e de mortalidade;

(iii) há uma concentração dos estudos nas regiões sul/sudeste e, apesar da importância do norte/nordeste tanto para alimentação quanto para reprodução de várias espécies, os dados ainda são escassos; e

(iv) considerando a carência de informações sobre as capturas pela pesca artesanal e sua grande diversificação ao longo da costa brasileira, se faz necessário melhor 
caracterizar e dimensionar as capturas e mortalidades conforme as diferentes pescarias e sua distribuição regional.

Na segunda linha, "compreender o impacto sobre as populações em termos de mortalidade de tartarugas e de alteração das comunidades marinhas" (Hamann et al., 2010), concluímos que:

(i) no caso do espinhel pelágico, os dados de CPUE existentes, apesar de não inferir diretamente a mortalidade, podem contribuir para o desenvolvimento de estratégias de conservação, considerando seu impacto sobre a estrutura das populações na costa brasileira; e

(ii) para todas as pescarias, industriais e artesanais, ainda não há uma clareza sobre a mortalidade das tartarugas e seu impacto nas populações, tão pouco as consequências para as comunidades marinhas.

Na terceira linha, "desenvolvimento de mudanças técnicas nos petrechos e a elaboração de estratégias políticas compatíveis com as necessidades socioeconômicas para reduzir os impactos do bycatch nas populações de tartarugas" (Hamann et al., 2010), concluímos que:

(i) as mudanças tecnológicas estão sendo desenvolvidas apenas para o espinhel-pelágico e focadas na avaliação da mudança de anzol, com inciativas ainda incipientes de outras mudanças para esta pescaria;

(ii) a despeito de algumas políticas voltadas para modificações em petrechos, as estratégias de ação mais comumente propostas na literatura abrangem sensibilização e educação ambiental de pescadores, mas com pouca avaliação de sua efetividade; e

(iii) ações envolvendo a participação de pescadores e outros stakeholders para monitoramento participativo e tomada de decisão colaborativa (i.e., arranjos de cogestão) são escassos.

A captura incidental na pesca artesanal, principalmente em redes de emalhe, ainda requer estudos que possam dimensionar a interação e avaliar seu impacto na mortalidade de jovens e adultos. Isto implica não somente em ampliar os estudos, mas também as estratégias de pesquisa e ações, tais como estudos de etnoconhecimento, monitoramentos participativos e engajamento em arranjos cogestão da pesca. Da mesma forma, ampliar os esforços de pesquisa para as regiões norte e nordeste, devido à carência de estudos em uma região com alta incidência de tartarugas marinhas e pescarias artesanais.

\section{REFERÊNCIAS BIBLIOGRÁFICAS}

Almeida, A.P.; Thomé, J.C.A.; Bellini, C.; Marcovaldi, M.A.; Santos, A. J.B.; Lopez, M. \& Santos, A.S. Avaliação do estado de conservação da tartaruga marinha Chelonia mydas (Linnaeus, 1758) no Brasil. Biodiversidade Brasileira, v. I, n. 1, p. 12-19, 2011a.

Almeida, A.P.; Thomé, J.C.A.; Baptistotte, C.; Marcovaldi, M.A.; Santos, A.S. dos \& Lopez, M. Avaliação do estado de conservação da tartaruga marinha Dermochelys coriacea (Vandelli, 1761) no Brasil. Biodiversidade Brasileira, v. I, n. 1, p. 37-44, 2011 b.

Armitage, D.R.; Plummer, R.; Berkes, F.; Arthur, R.I.; Charles, A.T.; Davidson-Hunt, I.J.; Diduck, A.P.; Doubleday, N.C.; Johson, D.S.; Marschke, M.; McConney, P.; Pinkerton, E.W. 
\& Wollenberg, E.K. Adaptive co-management for social-ecological complexity. Front. Ecol. Environ., v. 7, n. 2, p. 95-102, 2009.

Berkes, F.; Mahon, R.; McConney, P.; Pollnac, R. \& Pomeroy, R. Managing small-scale fisheries: alternative directions and methods. International Development Research Centre, Ottawa, Canada, 2001.

Biopesca - Projeto Biopesca. Captura Incidental. Disponível em: http:/ / www.biopesca.org. br/index.html. Acessado em: 21de novembro de 2015.

Braga, H.O. \& Schiavetti, A. Attitudes and local ecological knowledge of experts fishermen in relation to conservation and bycatch of sea turtles (reptilia: testudines), Southern Bahia, Brazil. J. Ethnobiol. Ethnomed., v. 9, n. 15, 2013.

Bunce, L.; Townsley, P.; Pomeroy, R. \& Pollnac, R. Socioeconomic manual for coral reef management. Australian Institute of Marine Science, 264 p., Townsville, Australia, 2000.

Campbell, L.M.; Silver, J.J.; Gray, N.J.; Ranger, S.; Brodericj, A.; Fisher, T.; Godfrey, M.H.; Gore, S.; Jeffers, J.; Martin, C.; McGowan, A.; Richardson, P.; Sasso, C.; Slade, L. \& Godley, B. Co-management of sea turtle fisheries: Biogeography versus geopolitics. Mar. Policy, v. 33, p. 137-145, 2009.

Castilhos, J; Coelho, C.A.; Argolo, J.F.; Santos, E.A.P.; Marcovaldi, M.A.; Santos, A.S. \& Lopez, M. Avaliação do estado de conservação da tartaruga marinha Lepidochelys olivacea (Eschscholtz, 1829) no Brasil. Biodiversidade Brasileira. v. I, n. 1, p. 28-36, 2011.

Castilhos, J. \& Tiwari, M. Preliminary data and observations from an increasing olive ridley population in Sergipe, Brazil. Mar. Turt. Newsl., v. 113, p. 6-7, 2006.

Dias, A.C.E.; Cinti, A. \& Seixas, C.S. Participatory Monitoring of Small-Scale Coastal Fisheries in Brazil and the Southern Cone: A Literature Review, in, Commons Amidst Complexity and Change, the Fifteenth Biennial Conference of the International Association for the Study of the Commons, May 25-29, Edmonton, Alberta, 2015.

Diegues, A.C. A pesca construindo sociedades. Núcleo de Apoio à Pesquisa Sobre Populações Humanas e Áreas Úmidas Brasileiras/USP, 315 p., São Paulo, 2004.

Fiedler, F.N.; Sales, G.; Giffoni, B. B.; Monteiro-Filho, E.L.A.; Secchi, E.R. \& Bugoni, L. Driftnet fishery threats sea turtles in the Atlantic Oceanic. Biodiversity Conservation, v, 21, p. 915-931, 2012.

Fiedler, F.N.; Port, D.; Giffoni, B.B.; Sales, G. \& Fisch, F. Pelagic longline fisheries in southeastern/south Brazil. Who cares about the law? Mar. Policy, v. 77, p. 56-64, 2017.

Gallo, B.M.G.; Macedo, S.; Giffoni, B.B.; Becker, J.H. \& Barata, P.C.R. Sea Turtle Conservation in Ubatuba, Southeastern Brazil, a Feeding Area with Incidental Capture in Coastal Fisheries. Chelonian Conserv. Bi., v. 5, n. 1, p. 93-101, 2006.

Galvão, M.C.; Moura, A.C.O.S. \& Monteiro, A. F. Educação Ambiental Através do Observador de Bordo: Uma Proposta para Inspirar Transformações. Rev. Eletrônica Mestr. Educ. Ambient., v. 24, 2010.

Guebert, F.M.; Rosa, L. \& Monteiro-Filho, E.L.A. Monitoramento da mortalidade de tartarugas marinhas no litoral paranaense, sul do Brasil, p. 50-52, in, Resumos da II Jornada de Conservação e Pesquisa de Tartarugas Marinhas no Atlântico Sul Ocidental, Rio Grande, RS, 2005. 
Hamann, M. et al. Global research priorities for sea turtles: informing management and conservation in the 21st century. Endanger. Species Res., v. 11, p. 245-269, 2010.

IUCN - International Union for Conservation of Nature. IUCN Red List of Threatened Species. Version 2013.1. <www.iucnredlist.org>. Acesso em 02 de Janeiro de 2018.

Kalikoski, D. C.; Seixas, C.S. \& Almudi, T. Gestão compartilhada e comunitária da pesca no Brasil: avanços e desafios. Ambiente E Sociedade, v. 12, p. 151-172, 2009

Lewinson, R.L.; Crowder, L.B.; Read, A.J. \& Freeman, S.A. Understanding impacts of fisheries bycatch on marine megafauna. Trends Ecol. Evol., v. 19, p. 598-604, 2004.

Lima, E.H.S.M.; Melo, M.T.D. \& Barata, P.C.R. Incidental capture of sea turtles by Lobster Fishery off the Ceará Coast, Brazil. Mar. Turt. Newsl., v. 128, p. 16-19, 2010.

Lima, E.H.S.M. \& Melo, M.T.D. Encalhes de tartarugas marinhas registradas pelo Projeto TAMAR-IBAMA ao longo do litoral do estado do Ceará, período 1996 - 2004, in, Resumos do II Congresso Brasileiro de Oceanografia, Vitória-ES, 2005.

López-Barrera, E.A.; Longo, G.O. \& Monteiro-Filho, E.L.A. Incidental capture of green turtle (Chelonia mydas) in gillnets of small-scale fisheries in the Paranaguá Bay, Southern, Brazil. Ocean Coast. Manage., v. 60, p. 11-18, 2012.

Malafaia, P.N.; Olavo, G.; França, A.R.; Seara, F.S.; Freitas, M.B.O.; Almeida, J.C. de; Alencar, S.M. de; Rêgo, L.S. \& Castro, M.S. de. Experiência de monitoramento participativo a bordo de embarcações da pesca artesanal no Território da Cidadania do Baixo Sul da Bahia, Brasil. Desenvolv. Meio Ambiente, v. 32, p. 165-180, 2014.

Marcovaldi, M.A.; Baptistotte, C.; Castilhos, J.C. de; Gallo, B.M.G.; Lima, E.H.S.M.; Sanches, T.M. \& Vieitas, C.F. Activities by Project TAMAR in Brazilian Sea Turtle Feeding Grounds. Mar. Turt. Newsl., v. 80, p. 5-7, 1998.

Marcovaldi, M.A. \& Chaloupka, M. Conservation status of the loggerhead sea turtle in Brazil: an encouraging outlook. Endang. Species Res., v. 3, p. 133-143, 2007.

Marcovaldi, M.A.; Lopez, G.G.; Soares, L.S.; Bellini, C.; Santos, A.J.B.; Lopez, M. \& Santos, A.S. Avaliação do estado de conservação da tartaruga marinha Eretmochelys imbricata (Linnaeus, 1766) no Brasil. Biodiversidade Brasileira, v. I, n. 1, p. 20-27, 2011.

Marcovaldi, M.A.; Lopez, G.G; Soares, L.S. \& Santos, A.J.B. Fifteen Years of Hawksbill Sea Turtle (Eretmochelys imbricata) Nesting in Northern Brazil. Chelonian Conserv. Bi., v. 6, 2007.

Marcovaldi, M.A. \& Marcovaldi, G.G. Marine turtles of Brazil: the history and structure of Projeto TAMAR-IBAMA. Biol. Conserv., v. 91, n. 1, p. 35-41, 1999.

Marcovaldi, M.A.; Sales, G.; Thomé, J.C.A.; Silva, A.C.C.D. da; Gallo, B.M.G.; Lima, E.H.S.M.; Lima, E.P. \& Bellini, C. Sea turtles and fisheries interactions in Brazil: identifying and mitigating potential conflicts. Mar. Turt. Newsl., v. 112, p. 4-8, 2006.

Marcovaldi, M.A.; Thomé, J.C.; Sales, G.; Coelho, C.A; Gallo, B. \& Bellini, C. Brazilian plan for reduction of incidental sea turtle capture in fisheries. Mar. Turt. Newsl., v. 96, p. 24-25, 2002.

Marcovaldi, M.A.; Lopez, G.G.; Soares, L.S.; Lima, E.H.S.M.; Thomé, J.C.A. \& Almeida, A.P. Satellite-tracking of female loggerhead turtles highlights fidelity behavior in northeastern Brazil. Endang. Species Res., v.12, p. 263-272, 2010 
Marcovaldi, M.A.; Lopez, G.G.; Soares, L.S. \& López-Mendilaharsuet, M. Satellite tracking of hawksbill turtles Eretmochelys imbricata nesting in northern Bahia, Brazil: turtle movements and foraging destinations. Endang. Species Res., v. 17, p. 123-132, 2012.

Monteiro, D.S.; Estima, S.C.; Gandra, T.B.R.; Silva, A.P.; Bugoni, L.; Swimmer, Y.; Seminoff, J.A. \& Secchi, E. R. Long-term spatial and temporal patterns of sea turtle strandings in southern Brazil. Mar. Biol., v. 163, n. 247, 2016.

Moore, J.E.; Coxa, T.M.; Lewisonn, R.L.; Read, A.J.; Bjorkland, R.; McDonal, S.L.; Crowder, L.B. \& Aruna, E. An interview-based approach to assess marine mammal and sea turtle captures in artisanal fisheries. Biol. Conserv., v. 143, p. 795-805, 2010.

Nagoaka, S.M.; Bondiolli, A.C.V. \& Monteiro-Filho, E.L.A. Sea turtle bycatch by Cercofixo in Cananéia lagoon estuarine complex, São Paulo, Brasil. Mar. Turt. Newsl., v. 119, p. 4-6, 2008.

Nogueira, M.M. \& Alves, R.R.N. Assessing sea turtle bycatch in Northeast Brazil through an ethnozoological approach. Ocean Coast. Manage., v. 133, p. 37-42, 2016.

NRC - National Research Council. Decline of sea turtles: causes and prevention. National Academy Press, Washington, DC. 1990.

Sales, G.; Giffoni, B. \& Barata, P.C.R. Incidental catch of sea turtles by the Brazilian pelagic longline fishery. J. Mar. Biol. Assoc. UK, v. 88, n. 4, p. 853-864, 2008.

Sales, G.; Giffoni, B.B.; Fiedler, F.; Azevedo, V.; Kotas, J.; Swimmer, Y. \& Bugoni, L. Circle hook effectiveness for the mitigation of sea turtle bycatch and capture of target species in a Brazilian pelagic longline fisher. Aquatic Conserv: Mar. Freshw. Ecosyst., v. 20, p. 428-436, 2010.

Samuel, S.; Morreale, J.; Clark, C.W.; Greene, C.H. \& Richmond, M.E. Underwater, low-frequency noise in a coastal sea turtle habitat. J. Acoust. Soc. Am., v. 117, n. 3, p. 1465-1472, 2005.

Santos, A.S.; Soares, L.S.; Marcovaldi, M.A.; Monteiro, D. S.; Giffoni, B. \& Almeida, A.P. Avaliação do Estado de Conservação da Tartaruga Marinha Caretta caretta Linnaeus, 1758 no Brasil. Biodiversidade Brasileira, v. I, n. 1, p. 3-11, 2011.

Silva, A.C.C.D; Castilhos, J.C.; Rocha, D.A.S.; Oliveira, F.L.C. \& Weber, M. Mortalidade de tartarugas marinhas no entorno de sítios de reprodução no Estado de Sergipe, Brasil, in, p. 453-454, Resumos 24th Congresso Brasileiro de Zoologia, Itajaí-SC, 2002.

Silva, A.C.C.D; Castilhos, J.C.; Lopez, G. \& Barata, P.C.R. Nesting biology and conservation of the olive ridley sea turtle (Lepidochelys olivacea) in Brazil, 1991/1992 to 2002/2003. J. Mar. Biol. Assoc. UK, v. 87, p. 1-10, 2007.

Silva, A.C.C.D.; Castilhos, J.C.; Santos, E.A.P.; Brondízio, L.S. \& Bugoni, L. Efforts to reduce sea turtle bycatch in the shrimp fishery. Ocean Coast. Manage., v. 53, p. 570-576, 2010.

Silva, A.C.C.D.; Santos, E.A.P.; Oliveira, F.L.C.; Weber, M.I.; Batista, J.A.F.; Serafini, T.Z. \& Castilhos, J.C. Satellite-tracking reveals multiple foraging strategies and threats for olive ridley turtles in Brazil. Mar. Ecol. Prog. Ser., v. 443, p. 237-247, 2011.

Soto, J.M.R.; Serafini, T.Z. \& Celini, A.A.O. Beach strandings of sea turtles in the state of Rio Grande do Sul: an indicator of gillnet interaction along the southern Brazilian coast, in, p. 276, Proceedings of the 22nd annual symposium on sea turtle biology and conservation, 2002. 
Thomé, J.C.A.; Baptistotte, C.; Moreira, L.M.P.; Scalfoni, J.T.; Almeida, A.P.; Riethi, D. B. \& Barata, P.C.R. Nesting Biology and Conservation of the LeatherbackSea Turtle(Dermochelys coriacea) in the State of Espírito Santo, Brazil, 1988-1989 to 2003-2004. Chelonian Conserv. Bi., v. 6, p.15-27, 2007. 


\section{APÊNDICE 1 \\ Lista da literatura sobre a Interação de Tartarugas Marinhas e a Pesca, publicadas entre 1965 e 2016, por estado/região}

\section{Águas Oceanicas}

Barceló, C.; Domingo, A.; Miller, P.; Ortega, L.; Giffoni, B.; Sales, G.; McNaughton, L.; Marcovaldi, M.; Heppell, S.S. \& Swimmer, Y. High-use areas, seasonal movements and dive patterns of juvenile loggerhead sea turtles in the Southwestern Atlantic Ocean. Mar. Ecol. Prog. Ser., v. 479, p. 235-250, 2013.

Bugoni, L.; Neves, T.S.; Leite Jr, N.O.; Carvalho, D.; Sales, G.; Furness, R.W.; Stein, C.E.; Peppes, F.V.; Giffoni, B.B. \& Monteiro, D.S. Potential bycatch of seabirds and turtles in hook-and-line fisheries of the Itaipava Fleet. Fish. Res., v. 90, p. 217-224, 2008.

Coelho, R.; Fernandez-Carvalho, J. \& Santos, M.N. A review of fisheries within the Iccat convention area that interact with sea turtle. Col. Vol. Sci. Pap. - Iccat., v. 69, n. 4, p. 1788$1827,2013$.

Domingo, A.; Sales, G.; Giffoni, B.B.; Miller, P.; Laporta, M. \& Maurutto, G. Captura incidental de tortugas marinas com palangre pelágico em el Atantico Sur por las flotas de Brasil y Uruguai. Col. Vol. Sci. Pap. - Iccat., v. 59, n. 3, p. 992-1002, 2006.

Fiedler, F.N.; Sales, G.; Giffoni, B. B.; Monteiro-Filho, E.L.A.; Secchi, E.R. \& Bugoni, L. Driftnet fishery threats sea turtles in the Atlantic Oceanic. Biodiversity Conservation, v, 21, p. 915-931, 2012.

Giffoni, B.; Domingo, A.; Sales, G.; Fiedler, F. N. \& Miller, P. Interacción de tortugas marinas (Caretta caretta y Dermochelys coriacea) con la pesca de palangre pelágico em el Atlántico Sudoccidental: uma perspectiva regional para la conservación. Col. Vol. Sci. Pap. - Iccat., v. 62, n. 6, p. 1861-1870, 2008.

Giffoni, B.; Leite Jr, N.; Miller, P.; Pons, M.; Sales, G. \& Domingo, A. Captura incidental de tortugas marinas por las flotas de palangre pelágico de Brasil y de Uruguai (1998 à 2010). Col. Vol. Sci. Pap. - Iccat., v. 70, n. 5, p. 2217-2225, 2014.

Kotas, J.E.; Gallo, B.M.G.; Santos, S.; Azevedo, V.G. \& Barata, P.C.R. Incidental captures of loggerheard (Caretta caretta) and leatherback (Dermochelys coriacea) sea turtles by the pelagic longline fishery off southern Brazil. Fish. B., v. 102, n. 2, p. 293-399, 2004.

Lewison, R.L.; Freeman, S.A. \& Crowder, L.B. Quantifying the effects of fisheries on threatened species: the impact of pelagic longlines on loggerheard and leatherback sea turtles. Ecol. Lett., v. 7, p. 221-231, 2004.

Lopez-Mendilaharsu, M.; Sales, G.; Giffoni, B.; Miller, P.; Fiedler, F. N. \& Domingo, A. Distribucion y Composicion de Tallas de Las Tortugas Marinas (Caretta caretta y Dermochelys coriácea) que interactuan con el palangre pelagico em el Atlantico Sur. Col. Vol. Sci. Pap. - Iccat., v. 60, n. 6, p. 2094-2109, 2007. 
Pacheco, J.C.; Kerstertter, D.W.; Hazin, H.; Segundo, R.S.S.L.; Graves, J.E.; Carvalho, F. \& Travassos, P.E. A comparison of circle hook and J hook performance in a western equatorial. Fish. Res., v. 101, p. 39-45, 2011.

Pinedo, M.C. \& Polacheck, T. Sea turtle by-catch in pelagic longline sets off southern Brazil. Biol. Conserv., v. 119, p. 335-339, 2004.

Pons, M.; Marroni, S.; Machado, I.; Ghattas, B. \& Domingo, A. Machine learning procedures: an application to by-catch of the marine turtles Caretta caretta in the southwestern Atlantico Ocean. Col. Vol. Sci. Pap. - Iccat., v. 64, n. 7, p. 2442-2454, 2009.

Pons, M.; Domingo, A.; Sales, G.; Fiedler, F. N.; Miller, P.; Giffoni, B. \& Ortiz, M. Standardization of CPUE of loggerhead sea turtle (Caretta caretta) caught by pelagic longliners in the Southwestern Atlantic Ocean. Aquat. Living Resour., v. 23, p. 65-75, 2010.

Sales, G.; Giffoni, B. \& Barata, P.C.R. Incidental catch of sea turtles by the Brazilian pelagic longline fishery. J. Mar. Biol. Assoc. UK, v. 88, n. 4, p. 853-864, 2008.

Sales, G.; Giffoni, B.B.; Fiedler, F.; Azevedo, V.; Kotas, J.; Swimmer, Y. \& Bugoni, L. Circle hook effectiveness for the mitigation of sea turtle bycatch and capture of target species in a Brazilian pelagic longline fisher. Aquatic Conserv: Mar. Freshw. Ecosyst., v. 20, p. 428-436, 2010.

Sampaio, C.L.S. Dermochelys coriacea (Leatherback Sea Turtle). Accidental capture. Herp. Review, v. 30, n. 1, 1999.

\section{Brasil}

Marcovaldi, M.A.; Thomé, J.C.; Sales, G.; Coelho, C.A; Gallo, B. \& Bellini, C. Brazilian plan for reduction of incidental sea turtle capture in fisheries. Mar. Turt. Newsl., v. 96, p. 24-25, 2002a.

Marcovaldi, G.; Marcovaldi, M.A.; Sales, G.; Thomé, J.C.; Coelho, A.C.; Gallo, B. Plano de ação nacional para a redução da captura incidental de tartarugas marinhas pela atividade pesqueira. Gerenciamento Costeiro Integrado, p. 36-37, $2002 \mathrm{~b}$.

Marcovaldi, M.A.; Sales, G.; Thomé, J.C.A.; Silva, A.C.C.D. da; Gallo, B.M.G.; Lima, E.H.S.M.; Lima, E.P. \& Bellini, C. Sea turtles and fisheries interactions in Brazil: identifying and mitigating potential conflicts. Mar. Turt. Newsl., v. 112, p. 4-8, 2006.

Santos, A.S.; Soares, L.S.; Marcovaldi, M.A.; Monteiro, D. S.; Giffoni, B. \& Almeida, A.P. Avaliação do Estado de Conservação da Tartaruga Marinha Caretta caretta Linnaeus, 1758 no Brasil. Biodiversidade Brasileira, v. I, n. 1, p. 3-11, 2011.

\section{Pará}

Brito, T.P.; Oliveira, A.N.D.; Silva, D.A.C. \& Rocha, J.A.S. Conhecimento ecológico e captura incidental de tartarugas marinhas em São João de Pirabas, Pará, Brasil. Biotemas, v. 28, n. 3, p. 159-175, 2015. 


\section{Ceará}

Lima, E.H.S.M.; Melo, M.T.D.; Godfrey, M.H. Incidental capture of Leatherback along the coast of Ceara, Brasil. Mar. Turt. Newsl., v. 117, p. 11, 2007.

Lima, E.H.S.M.; Melo, M.T.D. \& Barata, P.C.R. Incidental capture of sea turtles by Lobster Fishery off the Ceará Coast, Brazil. Mar. Turt. Newsl., v. 128, p. 16-19, 2010.

Paiva, M.P. \& Nomura, H. Sobre a Produção Pesqueira de Alguns Currais de Pesca do Ceará - Dados de 1962 a 1964. Arq. Est. Biol. Mar. Univ. Ceará, v. 5, n. 2, p. 175- 214, 1965.

\section{Paraiba}

Guebert, F.M.; Barletta, M. \& Costa, M.F. Threats to sea turtle populations in the Western Atlantic poaching and mortality in small-scale fishery gears. J. Coastal Res., v. 65, p. $42-47,2013$.

Nogueira, M.M. \& Alves, R.R.N. Assessing sea turtle bycatch in Northeast Brazil through an ethnozoological approach. Ocean Coast. Manage., v. 133, p. 37-42, 2016.

\section{Pernambuco}

Guebert, F.M.; Barletta, M. \& Costa, M.F. Threats to sea turtle populations in the Western Atlantic poaching and mortality in small-scale fishery gears. J. Coastal Res., v. 65, p. $42-47,2013$.

\section{Sergipe}

Silva, A.C.C.D.; Castilhos, J.C.; Santos, E.A.P.; Brondízio, L.S. \& Bugoni, L. Efforts to reduce sea turtle bycatch in the shrimp fishery. Ocean Coast. Manage., v. 53, p. 570-576, 2010.

\section{Bahia}

Braga, H.O. \& Schiavetti, A. Attitudes and local ecological knowledge of experts fishermen in relation to conservation and bycatch of sea turtles (reptilia: testudines), Southern Bahia, Brazil. J. Ethnobiol. Ethnomed., v. 9, n. 15, 2013.

\section{Espírito Santo}

Sales, G.; Marcovaldi, M.A. \& Giannini, F. Testing mitigation measures to reduce sea turtle incidental capture in longline fishing gear in Brazil. SWIMMER, 2006.

\section{Rio de Janeiro}

Sales, G.; Marcovaldi, M.A. \& Giannini, F. Testing mitigation measures to reduce sea turtle incidental capture in longline fishing gear in Brazil. SWIMMER, 2006.

\section{São Paulo}

Bahia, N.C.F. \& Bondioli, A.C.V. Interação das tartarugas marinhas com a pesca artesanal de cerco-fixo em Cananéia, litoral sul de São Paulo. Biotemas, v. 23, n. 3, p. 203-213, 2010. 
Damasio, L.M.A. \& Carvalho, A.R. Implications of consumption and ecological knowledge on the management of marine turtles on the Northern coast of São Paulo, Brazil. Bioikos, v. 24, n. 2, p. 95-104, 2010.

Gallo, B.M.G.; Macedo, S.; Giffoni, B.B.; Becker, J.H. \& Barata, P.C.R. Sea Turtle Conservation in Ubatuba, Southeastern Brazil, a Feeding Area with Incidental Capture in Coastal Fisheries. Chelonian Conserv. Bi., v. 5, n. 1, p. 93-101, 2006.

Nagoaka, S.M.; Bondiolli, A.C.V. \& Monteiro-Filho, E.L.A. Sea turtle bycatch by Cercofixo in Cananéia lagoon estuarine complex, São Paulo, Brasil. Mar. Turt. Newsl., v. 119, p. 4-6, 2008.

Sales, G.; Marcovaldi, M.A. \& Giannini, F. Testing mitigation measures to reduce sea turtle incidental capture in longline fishing gear in Brazil. SWIMMER, 2006.

Silva, G.C.; Vaz-dos-Santos, A.M. \& Maracini, P. Análise de encalhes de tartarugas marinhas (Testudines: Cheloniidae e Dermochelydae) nos municípios da baixada santista, Iguape e Cananéia no período de 2004 a 2011. Revista Ceciliana, v. 4, n. 2, p. 9-15, 2012.

Soares, D.B.R.; Silva, T.H.M.; Kamada, B.P. \& Giordano, F. Captura incidental de tartarugas marinhas na pesca artesanal nas praias: Asturias e Pereque - Municipio do Guarujá, SP Revista Ceciliana, v. 1, n. 2, p. 136-140, 2009.

\section{Paraná}

Guebert, F.M.; Barletta, M. \& Costa, M.F. Threats to sea turtle populations in the Western Atlantic poaching and mortality in small-scale fishery gears. J. Coastal Res., v. 65, p. 42-47, 2013.

López-Barrera, E.A.; Longo, G.O. \& Monteiro-Filho, E.L.A. Incidental capture of green turtle (Chelonia mydas) in gillnets of small-scale fisheries in the Paranaguá Bay, Southern, Brazil. Ocean Coast. Manage., v. 60, p. 11-18, 2012.

\section{Santa Catarina}

Guebert, F.M.; Barletta, M. \& Costa, M.F. Threats to sea turtle populations in the Western Atlantic poaching and mortality in small-scale fishery gears. J. Coastal Res., v. 65, p. 42-47, 2013.

Pupo, M.M.; Soto, J.M.R. \& Hanazaki, N. Captura incidental de tartarugas marinhas na pesca artesanal da Ilha de Santa Catarina, SC. Biotemas, v. 19, n. 4, p. 63-72, 2006.

\section{Rio Grande do Sul}

Monteiro, D.S.; Estima, S.C.; Gandra, T.B.R.; Silva, A.P.; Bugoni, L.; Swimmer, Y.; Seminoff, J.A. \& Secchi, E. R. Long-term spatial and temporal patterns of sea turtle strandings in southern Brazil. Mar. Biol., v. 163, n. 247, 2016.

Ramos, K.L. \& Vasconcellos, M.C. Characterization of the Interaction Between Sea Turtles and Bottom Gillnets in Southern Brazil Through Interviews with Fishers. Mar. Turt.

Newsl., v. 139, 2013. 